\title{
Electron and hole spin cooling efficiency in InAs quantum dots: The role of nuclear field
}

\author{
P. Desfonds, ${ }^{1}$ B. Eble, ${ }^{1}$ F. Fras, ${ }^{1}$ C. Testelin, ${ }^{1}$ F. Bernardot,${ }^{1}$ M. Chamarro,,${ }^{1, a)}$ \\ B. Urbaszek, ${ }^{2}$ T. Amand, ${ }^{2}$ X. Marie, ${ }^{2}$ J. M. Gérard, ${ }^{3}$ V. Thierry-Mieg, ${ }^{4}$ A. Miard, ${ }^{4}$ and \\ A. Lemaître ${ }^{4}$ \\ ${ }_{1}^{1}$ Institut des NanoSciences de Paris, CNRS-UMR 7588, UPMC Univ. Paris 06, 140 Rue de Lourmel, \\ F-75015 Paris, France \\ ${ }^{2}$ LPCNO, INSA, UPS, CNRS, Université de Toulouse, 135 Avenue de Rangueil, F-31077 Toulouse, France \\ ${ }^{3}$ CEA/INAC/SP2M, CNRS, UJF Nanophysics and Semiconductors, 17 Rue des Martyrs, 38054 \\ Grenoble Cedex 9, France \\ ${ }^{4}$ Laboratoire de Photonique et Nanostructures, CNRS, Route de Nozay, F-91460 Marcoussis, France
}

(Received 24 February 2010; accepted 21 March 2010; published online 28 April 2010)

\begin{abstract}
The spin dynamics of a resident carrier, hole or electron, in singly charged InAs/GaAs quantum dots has been measured by pump-probe experiments. The relative strength of the hole to the electron hyperfine couplings with nuclei is obtained by studying the magnetic-field dependence of the resident-carrier spin polarization. We find, in good agreement with recent theoretical studies, that the hole hyperfine coupling is ten times smaller than the electron one. (C) 2010 American Institute of Physics. [doi:10.1063/1.3394010]
\end{abstract}

The spin of a carrier confined in a semiconductor quantum dot (QD) is considered as a good candidate for realizing quantum bits in the solid state. The hyperfine interaction (HI) of an electron spin with the nuclei spins has been identified as the most efficient mechanism of electron spin relaxation or decoherence in QDs at low temperature. ${ }^{1-4}$ The electron HI has a Fermi-contact character, due to the s-type Bloch function of an electron, and is then expected to be much more efficient than the $\mathrm{HI}$ of a hole, which has a p-type Bloch function. Recent theoretical studies have shown that, for a hole spin, the dipole-dipole HI term is the dominant one and leads to an anisotropic $\mathrm{HI}$, in contrast to the isotropic electron HI. ${ }^{5,6}$ Moreover, the hole-nuclei interaction is far from being negligible and is predicted to be only one order of magnitude weaker than the electron-nuclei interaction in InAs QDs. Very recently, the first experimental evidences of the hole HI have been found, ${ }^{7,8}$ but its strength relative to the electron $\mathrm{HI}$ is a real question from an experimental point of view, whereas it is of prime importance for estimating not only decoherence times but also spin cooling rates. In this letter, we measure this relative strength by studying the magnetic-field dependence of the hole- and electron-spin polarizations obtained in p-doped and n-doped InAs QDs, respectively. Indeed, for both electron and hole, the HI can be modelized by a frozen effective nuclear field whose strength and direction vary from QD to QD. ${ }^{2,5,6}$ In the absence of an external magnetic field, each spin optically prepared orthogonally to the plane of QDs precesses coherently around the local nuclear field. The average carrier spin polarization in the QD ensemble partially decays with a characteristic initial time $T_{\Delta}^{e}\left(T_{\Delta}^{h}\right)$ for electrons (holes), as a consequence of the random distribution of the local nuclear effective fields. ${ }^{2,6}$ When an external field is applied in the out-of-the-plane direction, the effect of the $\mathrm{HI}$ on the carrier spin polarization can be strongly reduced if this external field is larger than the dispersion of the in-plane fluctuations of the effective nuclear

${ }^{\text {a)}}$ Electronic mail: maria.chamarro@insp.jussieu.fr. field. Then, the ensemble-average carrier-spin polarization shows a minimum in absence of an external field, and reaches a maximum for an applied magnetic field which depends on the HI strength. In such measurements, we obtain a good agreement with recent theoretical results.

The investigated samples are InAs/GaAs StranskiKrastanov structures grown by molecular beam epitaxy on a (001) GaAs substrate. The growth axis is denoted z. Two structures have been studied, containing p-doped or n-doped InAs QDs. They consist of 30 planes of InAs self-assembled QDs, separated by 38-nm-thick GaAs spacer layers. The QD areal density is about $10^{10} \mathrm{~cm}^{-2}$. A carbon delta-doping layer (nominal density $\sim 2 \times 10^{11} \mathrm{~cm}^{-2}$ ) located $2 \mathrm{~nm}$ below each QD layer was used to obtain p-doped QDs (sample I). The photoluminescence (PL) spectrum at $2 \mathrm{~K}$ is centered at $1.36 \mathrm{eV}$ and has a half width at half maximum (HWHM) of about $15 \mathrm{meV}$. The second structure was n-modulation doped $2 \mathrm{~nm}$ below each layer with a Si-dopant density equal to $6 \times 10^{10} \mathrm{~cm}^{-2}$ (sample II). The PL spectrum at $2 \mathrm{~K}$ shows a maximum at $1.32 \mathrm{eV}$ and a HWHM of about $25 \mathrm{meV}$.

The electron or hole spin initialization has been the center of several recent studies. ${ }^{7,9-12}$ Here we present pumpprobe experiments leading to all-optical initialization and read-out of resident carrier (hole or electron) spins. The photoinduced circular dichroism (PCD) has been measured by using a pump-probe experiment based on a picosecond mode-locked Ti:Sa laser described in Ref. 7. In order to avoid the build-up of a dynamic nuclear polarization, ${ }^{13}$ the pump beam polarization was $\sigma^{+} / \sigma^{-}$modulated at $42 \mathrm{kHz}$ with a photoelastic modulator.

A four-level system representing the lowest-lying energy levels of an InAs QD containing a single hole (electron) is shown in Figs. 1(a) and 1(b) [Figs. 2(a) and 2(b)], without and with an externally applied magnetic field. Several experiments have evidenced the mixed character of the hole states in self-assembled QDs (Refs. 14-16) which can arise from the anisotropic strain distribution in the QDs. Here, $\widetilde{\pi}$ and $\mathbb{I}$ denote the two mixed hole ground states. They can be 
(a) $\mathrm{B}=0$
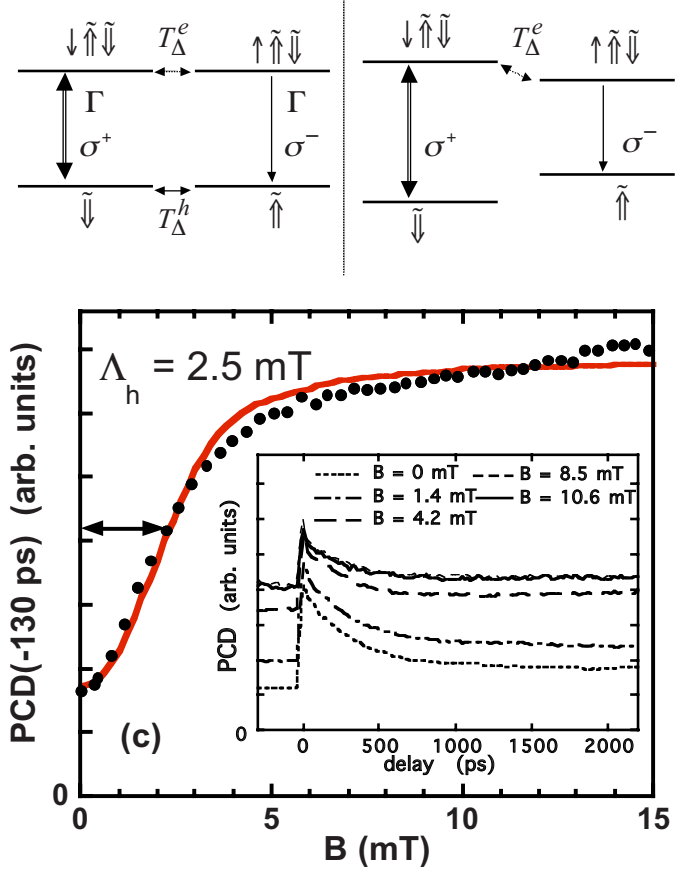

FIG. 1. (Color online) Level scheme of a p-doped QD (sample I) containing a single hole in the ground states and a positively charged trion in the excited states, for a Faraday magnetic field (a) B=0 and (b) $\Lambda_{h}<B<\Lambda_{e}$. The pump beam is $\sigma^{+}$circularly polarized. (c) Magnetic-field dependence of the PCD signal at negative delay and $T=2 \mathrm{~K}$ (dots) and theoretical fit with $T_{\Delta}^{h}=11 \mathrm{~ns}$ and $g_{h}=1.5$ for the hole, and $T_{\Delta}^{e}=800 \mathrm{ps}$ and $g_{e}=-0.8$ for the electron (solid line). Inset: Experimental PCD signal vs pump-probe delay, for different values of B.

written as linear combinations of pure heavy-hole (hh) $| \pm 3 / 2\rangle$ and light-hole (lh) $| \pm 1 / 2\rangle$ states, the relative weight of lh being $|\beta|^{2}{ }^{6,7} \uparrow$ and $\downarrow$ denote the electron ground states. $\uparrow \pi \widetilde{\Downarrow}$ and $\downarrow \pi \widetilde{\Downarrow}$ represent the positively charged trion states (the first excited states in p-doped QDs), and $\uparrow \downarrow \mathbb{\Downarrow}$ and $\uparrow \downarrow \Uparrow$ represent the negatively charged trion states in an n-doped QD. The mixed character of the hole states is at the origin of diagonal relaxation channels, as represented in Figs. 2(a) and 2(b) by a transition connecting the $\uparrow \downarrow \pi$ and $\downarrow$ states [a transition also exists between the $\uparrow \downarrow \mathbb{\Downarrow}$ and $\uparrow$ states, although not shown in Figs. 2(a) and 2(b)]. Such diagonal decays exist in p-doped QDs as well; they are not represented in Figs. 1(a) and 1(b), because they play a negligible role in the hole spin initialization. ${ }^{11,17}$

The spin initialization of the resident carriers can be obtained by an optical pulsed excitation, resonant with the trion transition.

In the case of p-doped QDs, after a $\sigma^{+}$-polarized excitation, a $\downarrow \pi \mathbb{\imath} \tilde{\downarrow}$ trion is created. The efficient electron-nuclei HI leads to a coherent coupling of both electron spin projections during trion lifetime $T_{R}=\Gamma^{-1}$, so that the $\downarrow \pi \widetilde{\Downarrow}$ can flip to state $\uparrow \pi \widetilde{I}$ [cf. Figs. 1(a) and 1(b)]. After emission of a circularly polarized $\sigma^{-}$photon, the resident spin is in state $\pi$, leading to a hole spin polarization. This hole spin cooling is particularly efficient if the hole HI which couples coherently the two hole spin ground states is a less efficient mechanism than the electron HI $\left(T_{\Delta}^{e} \ll T_{\Delta}^{h}\right)$, with comparable lifetime and electron dephasing time $\left(T_{R} \approx T_{\Delta}^{e}\right)$. (a) $\mathrm{B}=0$

(b) $\Lambda_{\mathrm{h}}<\Lambda_{\mathrm{e}}<\mathrm{B}$
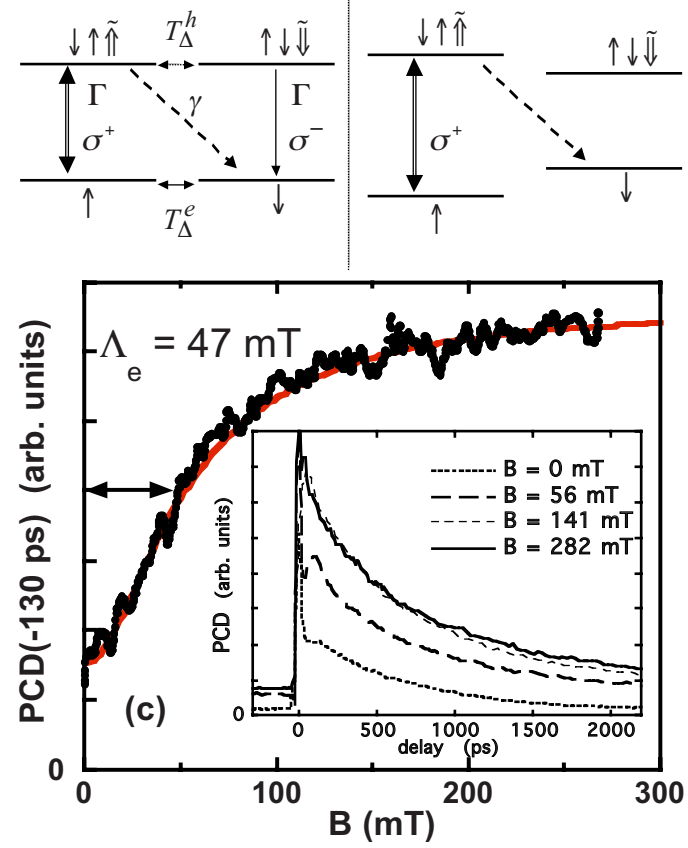

FIG. 2. (Color online) Level scheme of an n-doped QD (sample II) containing a single electron in the ground states and a negatively charged trion in the excited states, for a Faraday magnetic field (a) B=0 and (b) $\Lambda_{h}<\Lambda_{e}$ $<B$. The pump beam is $\sigma^{+}$circularly polarized. (c) Magnetic-field dependence of the PCD signal at negative delay and $T=2 \mathrm{~K}$ (dots) and theoretical fit with the same parameters of Fig. 1(c) (solid line). Inset: Experimental PCD signal vs pump-probe delay, for different values of B; the short-time oscillating signal is the contribution of the residual undoped QDs as discussed in Ref. 18.

The decay of the $(2 \mathrm{~K})$ PCD signal, obtained when the pump and probe beams are tuned at the trion transition of the p-doped QDs, is shown in the inset of Fig. 1(c). Without an applied magnetic field, we observe a nonzero PCD signal at negative pump-probe delays, indicating that the hole-spin polarization is not fully relaxed within the $T_{L}=13 \mathrm{~ns}$ repetition period of the laser. This long-living PCD signal is unambiguously associated to the spin polarization of the resident holes, since $T_{R}=800 \mathrm{ps},{ }^{7}$ and increases when a Faraday magnetic field is applied (i.e., the field is parallel to the propagation direction of the pump beam, along z). This applied field is low enough to negligibly screen the electronnuclei HI but high enough to screen the hole-nuclei interaction [cf. Fig. 1(b)]. Figure 1(c) shows the magnetic-field dependence of the spin polarization signal, i.e., the PCD signal at negative pump-probe delays. The HWHM is equal to $\Lambda_{h}=2.5 \mathrm{mT}$.

For n-doped QDs, under a circularly polarized $\sigma^{+}$pump, a trion $\downarrow \uparrow \pi$ is created [cf. Figs. 2(a) and 2(b)]. At zero field, two following processes are possible with comparable efficiency: (i) spin-flip between the $\pi$ and $\widetilde{\Downarrow}$ states via the hole $\mathrm{HI}$, and (ii) diagonal transitions associated to the hole mixing, with an emission rate $\gamma$. Compared to the p-doped QDs, the initialization of the resident electrons in n-doped QDs does need the participation of a diagonal transition. ${ }^{9}$ The inset of Fig. 2(c) shows the time-dependence of the PCD signal obtained when the pump and probe beams are tuned at the trion transition of the n-doped QDs. ${ }^{18}$ We underline that, in contrast to the p-doped QDs, the PCD signal at negative delays is nearly equal to zero without magnetic field. This 
means that the initialization of the electron spin is not an efficient process at zero field because the electron $\mathrm{HI}$ leads to a very efficient dephasing mechanism $\left(T_{\Delta}^{e} \ll T_{L}\right){ }^{3}$ Figure $2(\mathrm{c})$ shows the magnetic-field dependence of the PCD signal at negative delays. Though the signal shape is very similar to the one obtained for p-doped QDs, note the very different field scales in Figs. 1(c) and 2(c). Figure 2(c) with a HWHM equal to $\Lambda_{e}=47 \mathrm{mT}$, shows that the spin initialization of resident electrons is achieved, as in Ref. 9, when a Faraday magnetic field B is applied. As discussed before, such a field screens the hole spin HI $\left(B \gg \Lambda_{h}\right)$ so that the electron spin initialization is only induced by the diagonal transition.

A quantitative determination of the relative strength of the electron and hole $\mathrm{HI}$ can be obtained by using recent models which describe the interconnected hole and electron spin dynamics in p- or n-doped QDs, under a periodic pulsed excitation. $^{17,19}$ The PCD signal at negative delay is proportional to the resident spin polarization. For p-doped QDs, hh-lh mixing is relevant for the hole spin relaxation processes and has been neglected in the optical selection rules due to its minor contribution. For n-doped QDs, due to the role of the diagonal transition, we took into account all the optical transitions allowed by the hh-lh mixing. Note that the field dependence of the PCD signal at negative delays is, mainly, determined by the hh-lh mixing and resident spin parameters as the dephasing time and the Landé factor. We took a typical value of $|\beta| \approx 0.4$, as estimated recently in the literature. ${ }^{14-16}$ By using the mentioned models, we have fitted the PCD signal at negative delay versus magnetic field for both samples [cf. Figs. 1(c) and 2(c)] keeping the same parameters. The fitting parameters are, respectively, $T_{\Delta}^{h}$ $=11 \mathrm{~ns}$ and $g_{h}=1.5$ for the hole, and $T_{\Delta}^{e}=800 \mathrm{ps}$ and $g_{e}=$ -0.8 for the electron. One can remark that the relation between the experimental HWHM $\Lambda_{e}$ and $\Lambda_{h}$ and the carrier spin parameters, $\left(\left|g_{e}\right| \Lambda_{e} T_{\Delta}^{e}\right) /\left(g_{h} \Lambda_{h} T_{\Delta}^{h}\right) \approx 1$, is verified, in agreement with the frozen nuclear field model. Moreover, from the values of the dephasing times, one estimates the HI strength ratio $T_{\Delta}^{h} / T_{\Delta}^{e}=14$ in agreement with theoretical calculations. $^{6}$

In conclusion, we have performed pump-probe experiments in p-doped and n-doped QDs leading to the initialization and the read out of the resident carrier spins. The initialization of hole spins is accomplished via the electron-nuclei hyperfine coupling. By comparison, the initialization of resident electrons is clearly obtained only when an external magnetic field is applied in Faraday configuration, and needs the presence of a quasiforbidden diagonal transition associated to hole mixing. The study of the magnetic-field dependence of the carrier spin polarization gives us the relative strength of the hole to the electron hyperfine couplings, which is in good agreement with recent theoretical studies.

We acknowledge support from the French ANR-P3N contract QUAMOS number ANR-09-NANO-030-04 and region ile de France.

${ }^{1}$ A. V. Khaetskii, D. Loss, and L. Glazman, Phys. Rev. Lett. 88, 186802 (2002); Phys. Rev. B 67, 195329 (2003).

${ }^{2}$ I. A. Merkulov, A. L. Efros, and M. Rosen, Phys. Rev. B 65, 205309 (2002).

${ }^{3}$ P. F. Braun, X. Marie, L. Lombez, B. Urbaszek, T. Amand, P. Renucci, V. K. Kalevich, K. V. Kavokin, O. Krebs, P. Voisin, and Y. Masumoto, Phys. Rev. Lett. 94, 116601 (2005).

${ }^{4}$ A. C. Johnson, J. R. Petta, J. M. Taylor, A. Yacoby, M. D. Lukin, C. M. Marcus, M. P. Hanson, and A. C. Gossard, Nature (London) 435, 925 (2005).

${ }^{5}$ J. Fischer, W. A. Coish, D. V. Bulaev, and D. Loss, Phys. Rev. B 78, 155329 (2008).

${ }^{6}$ C. Testelin, F. Bernardot, B. Eble, and M. Chamarro, Phys. Rev. B 79, 195440 (2009).

${ }^{7}$ B. Eble, C. Testelin, P. Desfonds, F. Bernardot, A. Balocchi, T. Amand, A. Miard, A. Lemaître, X. Marie, and M. Chamarro, Phys. Rev. Lett. 102, 146601 (2009).

${ }^{8}$ H. Kurtze, D. R. Yakovlev, D. Reuter, A. D. Wieck, and M. Bayer, arXiv:0905.1586v1 (unpublished).

${ }^{9}$ M. Atature, J. Dreiser, A. Badolato, A. Hogele, K. Karrai, and A. Imamoglu, Science 312, 551 (2006).

${ }^{10}$ X. Xu, Y. Wu, Q. Bo, S. Huaang, J. Chen, D. G. Steel, A. S. Bracker, D Gammon, C. Emary, and L. Sham, Phys. Rev. Lett. 99, 097401 (2007).

${ }^{11}$ B. D. Gerardot, D. Brunner, P. A. Dalgarno, P. Ohberg, S. Seidl, M. Kroner, K. Karrai, N. G. Stoltz, P. M. Petroff, and R. J. Warburton, Nature (London) 451, 441 (2008).

${ }^{12}$ A. J. Ramsay, S. J. Boyle, R. S. Kolodka, J. B. B. Oliveira, J. SkibaSzymanska, H. Y. Liu, M. Hopkinson, A. M. Fox, and M. S. Skolnick, Phys. Rev. Lett. 100, 197401 (2008).

${ }^{13}$ P. Maletinsky, A. Badolato, and A. Imamoglu, Phys. Rev. Lett. 99, 056804 (2007).

${ }^{14}$ D. N. Krizhanovskii, A. Ebbens, A. I. Tartakovskii, F. Pulizzi, T. Wright, M. S. Skolnick, and M. Hopkinson, Phys. Rev. B 72, 161312 (2005)

${ }^{15}$ A. V. Koudinov, I. A. Akimov, Yu. G. Kusrayev, and F. Henneberger, Phys. Rev. B 70, 241305 (2004).

${ }^{16}$ Y. Léger, L. Besombes, L. Maingault, and H. Mariette, Phys. Rev. B 76, 045331 (2007).

${ }^{17}$ B. Eble, P. Desfonds, F. Fras, F. Bernardot, C. Testelin, M. Chamarro, A. Miard, and A. Lemaître, Phys. Rev. B 81, 045322 (2010).

${ }^{18}$ F. Bernardot, E. Aubry, J. Tribollet, C. Testelin, M. Chamarro, L. Lombez, P.-F. Braun, X. Marie, T. Amand, and J.-M. Gérard, Phys. Rev. B 73, 085301 (2006).

${ }^{19}$ I. A. Yugova, A. A. Sokolova, D. R. Yakovlev, A. Greilich, D. Reuter, A. D. Wieck, and M. Bayer, Phys. Rev. Lett. 102, 167402 (2009). 